\title{
Rice Kernel Shape Description Using an Improved Fourier Descriptor ${ }^{*}$
}

\author{
Hua Gao, Yaqin Wang ${ }^{* *}$, Guangmei Zhang, Pingju Ge, and Yong Liang \\ College of Information Science \& Engineering, Shandong Agricultural University, \\ No. 61 Daizong Road, Taian, Shandong 271018, China \\ \{wyq, gaoh $\}$ asdau. edu.cn
}

\begin{abstract}
A Fourier descriptor is one of the best methods for describing object boundaries, but there are limitations in describing the boundary of rice kernels using traditional Fourier descriptors. An innovative approach was developed to describe rice kernel boundaries by improving a traditional Fourier descriptor. This radius Fourier descriptor (RFD) uses a radius set for rice kernel images as its basis function, and uses amplitude spectrum of Fourier transform for the radius set as its descriptor. This method only retains the first 9 components of RFD, which is simple and the dimension of the feature vector can be reduced greatly without concern for the initial starting point on the contour. The method was validated in terms of area computation, variety distance calculation, shape description, and detection of broken kernels using a backpropagation (BP) neural network for several varieties of rice kernels. The detection accuracy for whole rice kernels of different samples was $96 \%-100 \%$ and for broken rice kernels was $96.5 \%$.
\end{abstract}

Keywords: Image processing, Radius set, Rice kernel shape description, Radius Fourier descriptor.

\section{Introduction}

The shape of rice kernels is a basic parameter for quality assessment and classification of rice[1-2]. According to GB 1354-1986, the main parameters for rice quality assessment include machining precision, grain integrity, impurities, and broken rice rate. These parameters are related to kernel shape directly or indirectly. To realize detection of rice parameters using machine vision, one of the major challenges is to find a method to effectively and accurately describe rice shape features and discriminate whole rice kernels. At present, there are several parameters used to describe the kernel shape of agricultural products[3-4], including area, eccentric

\footnotetext{
Supported by the Innovation and Technology Fund of Shandong Agricultural University of China under Grant No.23660. First author: Hua Gao, 1963, Male, Professor. Research interests: Image processing, Precision agriculture.

** Corresponding author.
} 
moment, elongation, inertial center, aspect ratio, flakiness ratio, sphericity, roundness and Fourier descriptors. While for shape-based pattern recognition technique, moment invariant[5] and Fourier descriptor [6-8] are two major image recognition methods.

Experimental tests indicated that coordinate sequences for object contours based on a Fourier descriptor had the best shape recognition ability. Kauppien and Sepanen compared auto regressive and Fourier based descriptors in 2D shape classification [6]. Wei et al. reviewed on shape representation techniques and their applications in image retrieval[9]. Zhang and Lu classified and reviewed some important shape representation and description techniques[10]. However, traditional descriptors use Fourier transforms of a series of coordinate pairs for a shape boundary. According to the properties of the Fourier transform, a traditional Fourier descriptor is related to boundary scale, direction, and starting position of the curve, resulting in many limitations in describing rice shape boundaries. According to the periodicity of Fourier transform, when the original function of a Fourier transform shifts, the phase spectrum of Fourier transform will change, but the amplitude spectrum of Fourier transform will not change. In this paper, the amplitude of Fourier transform coefficient for a boundary radius set is used as a descriptor to describe rice kernel shape. This descriptor is named as Radius Fourier Descriptor (RFD). The rotation of rice kernel, corresponding to the shift of radius set, affects phase spectrum only, but does not affect amplitude spectrum. Therefore, it is rotation invariant to describe rice kernel shape using the RFD.

\section{Materials and Methods}

\subsection{Image Acquisition}

Several varieties of whole rice kernels and broken ones, including Dongbei long grain rice, Meihe rice, and Thai jasmine rice were taken as experimental objects. The original rice kernel images were acquired on a fixed image collection bench in this research. The surface of the image collection bench was covered with black cloth. A lighting chamber comprising a metal cylinder of $60 \mathrm{~cm}$ in diameter and $60 \mathrm{~cm}$ in height contained four $10-\mathrm{W}$ ordinary fluorescent lamps installed symmetrically. The internal wall of the chamber was painted white to yield equal diffuse reflection. The test camera (Canon A580), fixed with a homemade arm with adjustable position and height, had a resolution of $3264 \times 2448$ and was placed at the center of the chamber, 50 $\mathrm{cm}$ above the bench. Part of the original rice kernel images were shown in Fig. 1. To determine the boundary of every rice kernel, several preprocessing steps including image filtering, binarization and segmentation[11] were carried out. Fig. 2 was the segmentation result of Fig. 1a. Based on the segmentation result, the boundary of every rice kernel was obtained. Fig. 3 was the boundary image of five touched kernels on the top-left corner of Fig. 2. 


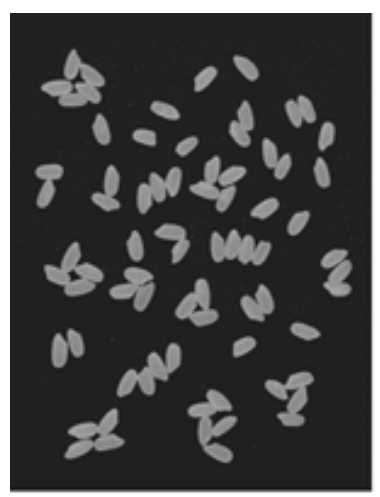

a

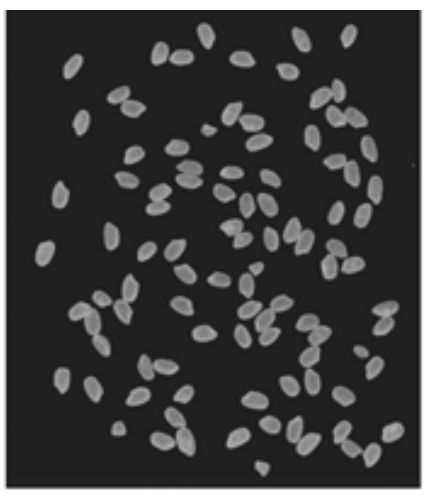

b

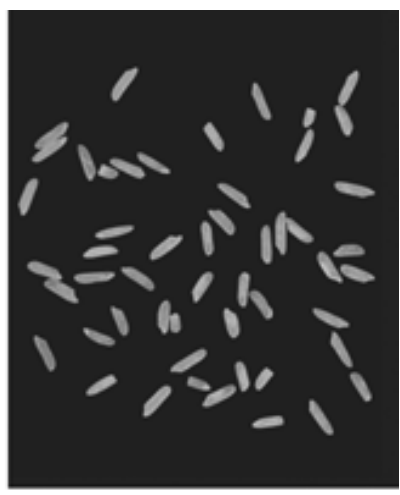

c

Fig. 1. Rice kernel images: (a) Dongbei long grain rice; (b) Meihe rice; (c) Thai jasmine rice

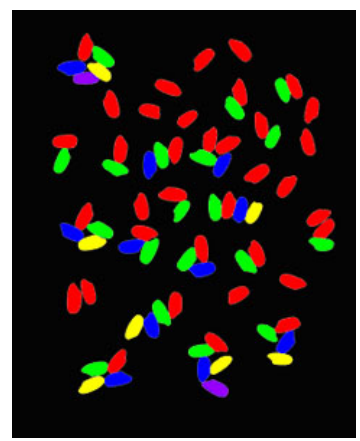

Fig. 2. The segmentation result of Fig. 1a

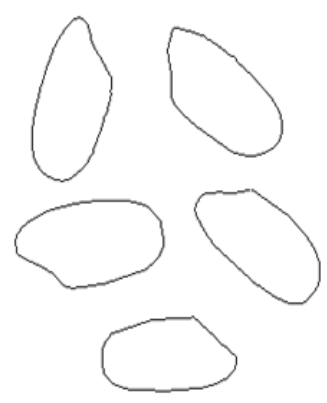

Fig. 3. The boundary of the five touched kernels on the top-left corner of Fig. 2

\subsection{Fourier Descriptor}

Position information for boundary points is vital in defining image boundaries, whereas gray information can be neglected in this study. A normalized boundary is a closed curve, so the boundary can be regarded as a curve $y=f(x)$ consisting of a set of points in the regular $\mathrm{x}-\mathrm{y}$ Cartesian coordinate system. A large amount of data is needed to totally describe such a curve in the spatial domain, resulting in complicated feature extraction. Thus, a boundary can be described in the frequency domain using a Fourier transform in a method known as a Fourier descriptor[8]. Traditional Fourier descriptor is related to boundary scale, direction, and start point position of curve, which result in many limitations for describing rice boundary with traditional Fourier descriptor. The amplitude of Fourier transform coefficient for a boundary radius set is used as a descriptor to describe rice kernel shape. This method can describe rice kernel shape accurately, and it is rotation invariant. Without concern for the start point of boundary when calculating, the method is simple to process and has a small amount of data. 
If Fourier transformation is performed using $y=f(x)$ directly, the transform result will depend on the specific coordinates of $x$ and $y$, which cannot satisfy shift and rotation invariability requirements[12]. Thus, the RFD was introduced to satisfy these requirements.

Definition 1. Suppose that a closed image boundary is a set $B$ consisting of $M$ boundary points and $b_{j}\left(x_{j}, y_{j}\right)$ is any point on the boundary. The center point of the boundary, $p_{\mathrm{o}}\left(x_{\mathrm{o}}, y_{\mathrm{o}}\right)$, can then be defined as:

$$
x_{\mathrm{o}}=\sum_{j=0}^{M-1} x_{j} / M, \quad y_{\mathrm{o}}=\sum_{j=0}^{M-1} y_{j} / M \quad, \quad b_{j}\left(x_{j}, y_{j}\right) \in B .
$$

Definition 2. As shown in Fig. 4, $N$ radial lines were constructed from $p_{o}$ to object image boundary with equal angle interval of $2 \pi / \mathrm{N}$. Let $b_{i}\left(x_{i}, y_{i}\right)$ be the intersection point of the radial lines and the object boundary, where $i=0,1,2, \ldots, N-1$. The radius descriptor of the object can then be defined as radius set $R=\left[r_{0}, r_{1}, \ldots r_{N-1}\right]$, where:

$$
r_{i}=\sqrt{\left(x_{\mathrm{o}}-x_{i}\right)^{2}+\left(y_{\mathrm{o}}-y_{i}\right)^{2}} \text {. }
$$

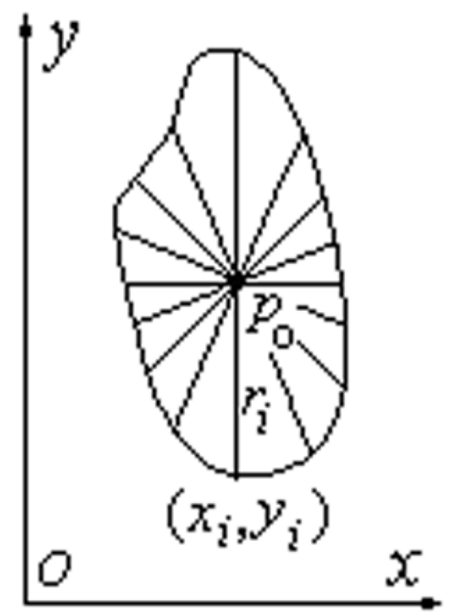

Fig. 4. Diagram showing calculation of the radius descriptor $R$

Because of the rigidity characteristic of rice kernel, it is impossible to produce deep hole on the fracture surface of rice kernel after breaking. Therefore, radial lines from the mass center intersect a rice boundary only once either for intact grains or for broken ones.

Definition 3. The discrete Fourier transform for $R$ is described by the equation:

$$
F_{r}(u)=\frac{1}{\sqrt{N}} \sum_{i=0}^{N-1} r_{i} \exp \left[-j \frac{2 \pi u i}{N}\right]
$$




$$
\left|F_{r}(u)\right|=\sqrt{R^{2}(u)+I^{2}(u)}
$$

Where $j^{\wedge} 2=-1 . R(u)$ is the real part of $\operatorname{Fr}(u)$, and $I(u)$ is the imaginary part of $\operatorname{Fr}(u)$. And $\left|F_{r}(u)\right|$ is defined as the RFD of the object boundary. According to the process of FFT algorithm, the value of $N$ is 2 to the power of $\mathrm{n}$ ( $\mathrm{n}$ is an integer). It is usually not necessary to represent rice kernel shape with very high precision in practical applications, so the value of $N$ is not necessary to be too big (the bigger the value of $N$, the more accurate the description). Experimental test indicated that 32 or 64 of $N$ can express the detail of rice kernel shape accurately. The value of $N$ is set to 32 in this research.

According to the periodicity of Fourier transform, when the original function of a Fourier transform shifts, the phase spectrum of Fourier transform will change, but not the amplitude spectrum. Therefore, the amplitude spectrum of RFD is rotation invariant.

\subsection{RFD Calculation}

The first step in RFD calculation is to find the boundary center point $p_{o}$ and then the intersection point $b_{i}\left(x_{i}, y_{i}\right)$ of the radial lines and the boundary, with $2 \pi / N$ as the interval angle. Because of the discreteness of digital images, many radial lines might not intersect with the boundary so the coordinates of a virtual intersection point coordinates must be obtained by interpolation. The specific algorithm proposed in this paper is as follows.

(1) Rice image boundary: The original rice image was processed via filtering, segmentation and binarization to obtain a binary image. Then the 8-neighbour boundary of the rice kernel was obtained using a morphological method:

$$
B=A-(A \Theta E)
$$

Where $A$ is the binary image, $E=\left|\begin{array}{lll}0 & 1 & 0 \\ 1 & 1 & 1 \\ 0 & 1 & 0\end{array}\right|$ is a structural erosion element, and operator "- " stands for the subtraction operation, and " $\Theta$ " stands for the erosion operation.

(2) Coordinates of the boundary center $p_{0}$ : For any point on the boundary as the starting point, each point was traversed along the boundary from the start until the point was reached again. The average of all traverse points was calculated using Eq. (1) to yield the coordinates of $p_{\mathrm{o}}\left(x_{\mathrm{o}}, y_{\mathrm{o}}\right)$.

(3) Similar circle radius:

(i) Find the first boundary point $b_{0}$ along the straight line $y=x_{\mathrm{o}}$ from $p_{\mathrm{o}}\left(x_{\mathrm{o}}\right.$, $\left.y_{\mathrm{o}}\right)$ and traverse every point $b_{j}\left(x_{j}, y_{j}\right)$ on the boundary to calculate the angle $\varphi_{j}$ : 


$$
\phi_{j}=\arccos \left(\frac{\left|x_{j}-x_{o}\right|}{\sqrt{\left(x_{j}-x_{o}\right)^{2}+\left(y_{j}-y_{o}\right)^{2}}}\right)
$$

(ii) Confirm an intersection point for the radius and boundary, $b_{i}$ $(i=0,1,2, \ldots N-1)$. If $\varphi_{j}=i^{*} 2 \pi / N$, point $b_{j}$ is an intersection point for a similar circle radius and the boundary. If $\varphi_{j}<i^{*} 2 \pi / N$, find the next point $b_{j+1}$. If $\varphi_{j}>i^{*} 2 \pi / N$, the coordinates of $b_{i}$ can be calculated according to:

$$
\begin{aligned}
& x_{i}=x_{j-1} \times \frac{2 \pi i / N-\phi_{j-1}}{\phi_{j}-\phi_{j-1}}+x_{j} \times \frac{\phi_{j}-2 \pi i / N}{\phi_{j}-\phi_{j-1}} \\
& y_{i}=y_{j-1} \times \frac{2 \pi i / N-\phi_{j-1}}{\phi_{j}-\phi_{j-1}}+y_{j} \times \frac{\phi_{j}-2 \pi i / N}{\phi_{j}-\phi_{j-1}}
\end{aligned}
$$

(iii) Obtain $R$ by calculating $r_{i}$ according to Eq. (2).

(4) Fourier transform for $R$ : According to Eq. (3), all calculations require $N^{2}$ multiplications and $N(N-1)$ additions so the computational workload increases greatly with $N$. To improve the computational efficiency, a fast Fourier transform (FFT) was used for practical application:

$$
\left[\begin{array}{c}
F_{r}(0) \\
\vdots \\
F_{r}(N-1)
\end{array}\right]=\left[\begin{array}{ccc}
w_{0,0} & \cdots & w_{0, N-1} \\
\vdots & \ddots & \vdots \\
w_{N-1,0} & \cdots & w_{N-1, N-1}
\end{array}\right]\left[\begin{array}{c}
r_{0} \\
\vdots \\
r_{N-1}
\end{array}\right]
$$

Where $w_{i, u}=\frac{1}{\sqrt{N}} e^{-j 2 \pi \frac{i u}{N}}$.

The computational complexity can be reduced to $\operatorname{Nog}_{2}(N)$ when using FFT for $N=2^{n}$, otherwise zero should be added.

\subsection{RFD Intercept}

The Fourier transform is a reversible linear integral transform (Eqs. (3) and (4)). Therefore, the radius descriptor $r_{i}$ can be restored by the Fourier descriptor $F_{r}(u)$. If all the coefficients of the Fourier transform were chosen as the boundary description feature, the dimension of the feature vector would be too high and the computational workload would be excessive, even though no contour information would be lost. Such high precision is usually not necessary in practical applications. Therefore, selective interception for the Fourier transform coefficient is essential. 
$F_{r}(u)$ is a sequence of $N$ frequency points and has conjugate symmetry with Nyquist frequency $n=N / 2+1$ as its center. Therefore, only half of the $F_{r}(u)$ points $(N / 2)$ are required for spectral analysis.

Because the power of Fourier spectrum mainly focuses on the low-frequency components, the fundamental shape of rice kernel can be described with small amount of low-frequency components. Experiments have been done to test how many components should be reserved to restore the rice kernel shape. Fig.5 shows the restoration of rice kernel shape with different number of components, such as $3,5,7$, and 9 components. Test result indicated that the first 9 components of RDF could restore the contours of object almost without distortion[13]. Therefore, only the first 9 components of RFD (that is the first 9 low-frequency coefficients of DFT) are required for rice shape description.

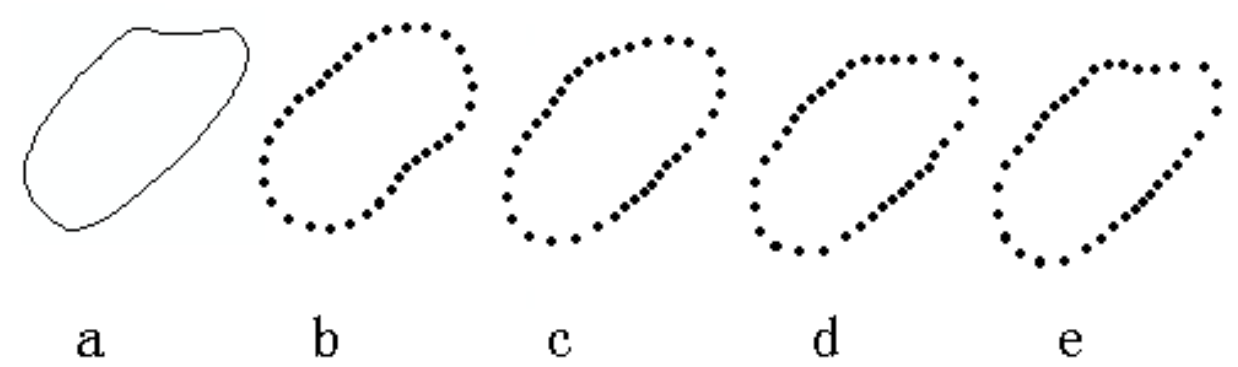

Fig. 5. Diagram showing restoration of the rice shape with different number of Fourier lowfrequency components: (a) original image; (b) restoration with 3 components; (c) restoration with 5 components; (d) restoration with 7 components; and (e) restoration with 9 components

\section{$3 \quad$ Results and Analysis}

According to the physical meaning of Fourier transform, each component of the descriptor represents a specific meaning of rice shape. That is, $F_{r}(0)$ includes the scale information, $F_{r}(1)$ includes the circularity information, $F_{r}(2)$ includes the length information, $F_{r}(3)$ includes the triangularity information, $F_{r}(4)$ includes the rectangularity information, $F_{r}(5)$ includes the pentagon information, $F_{r}(6)$ includes the hexagon information, and so on. Each kind of information can be used to perform shape calculation and recognition for different kind of applications. For example, $F_{r}(0)$ could calculate area approximately, and the first 9 components could detect rice variety and broken rice kernel. Especially, with the development of neural network, reliable classification and recognition can be finished only through appropriate discipline without regard for the meaning of components of Fourier descriptor.

To verify the usability of the RFD, experiments were carried out on some whole rice kernels and broken ones of China Dongbei long grain rice, Meihe rice and Thai jasmine rice. Results for kernel area, variety distance computation, and rice variety and broken kernel detection using RFD are discussed in the following sections. 


\subsection{Computation of Kernel Area}

According to the characteristic of Fourier transform, the first coefficient of Fourier transform reflects the scale information of image. If we take the rice kernel image as a similar circle, we can use $r_{a}=F_{r}(0) / N$ representing rice kernel radius approximately. Therefore, we can use the formula $S=k \pi r_{a}^{2}$ to approximately express the area of the enclosed region, where $k$ is an adjustment coefficient that can be set to different values for different objects according to experience. Table 1 lists the kernel area results for sample rice kernels. The actual area was obtained using a pixel statistical method. The unit of measurement would be size instead of pixels in practical applications.

Table 1. Calculation result of rice kernel area

\begin{tabular}{lccccc}
\hline \multirow{2}{*}{ Sample } & \multirow{2}{*}{ Number } & \multicolumn{5}{c}{ Average area (pixels) } \\
\cline { 3 - 7 } & & Calculated & Actual & Error & STDEV \\
\hline Broken kernels & 50 & 2538 & 2446 & 238 & $213(8.71 \%)$ \\
$\begin{array}{l}\text { Whole kernels of Dongbei } \\
\text { long grain rice }\end{array}$ & 50 & 4636 & 4600 & 194 & $205(4.46 \%)$ \\
$\begin{array}{l}\text { Whole kernels of Meihe rice } \\
\begin{array}{l}\text { Whole kernels of Thai } \\
\text { jasmine rice }\end{array}\end{array}$ & 50 & 4516 & 4497 & 167 & $151(3.36 \%)$ \\
\hline
\end{tabular}

\subsection{Shape Similarity}

The basis function of the Fourier transform is a set of orthogonal normalization functions. Its transform coefficient is determined by the inner product of the input function (image) and its basis function and is a measure of the similarity of the input and basis functions. Therefore, the distance for RFD spectral vectors can be used to measure the similarity of two contours. To compare rice kernels of different size and resolution, a normalization procedure should be applied to $F_{r}(u)$ according to:

$$
F_{r s}(u)=\frac{F_{r}(u)}{r_{a}}
$$

The normalized RFD is scale invariance and is only associated with the contour shape and is not related to the size of the rice kernel. Therefore, the distance between the normalized Fourier spectrum vectors for two contours can be used as variety distance for rice variety and broken kernel classification and differentiation. For easy classification, the Euclidean distance $\delta\left(x_{k}, x_{l}\right)$ is used to describe the contour similarity for rice kernels $x_{k}$ and $x_{l}$ :

$$
\delta\left(x_{k}, x_{l}\right)=\left[\sum_{i=1}^{m}\left(\left|F_{r s k}(i)\right|-\left|F_{r s l}(i)\right|\right)^{2}\right]^{\frac{1}{2}}
$$


Where $m$ is the number of components of the Fourier descriptor. The smaller the $\delta\left(x_{k}\right.$, $x_{l}$ ), the smaller the difference between two rice kernels and the greater their similarity. If $\delta\left(x_{k}, x_{l}\right)=0$, the two kernels are identical. Data on shape similarity for different rice samples are listed in Table 2.

Table 2. Shape similarity for rice kernels

\begin{tabular}{lccc}
\hline Comparison & Number & Average similarity & Variance \\
\hline $\begin{array}{l}\text { Whole Dongbei long grain rice } \\
\text { kernels }\end{array}$ & 50 & 0.027790 & 0.000558 \\
$\begin{array}{l}\text { Whole Meihe rice kernels } \\
\text { Whole Thai jasmine rice kernels }\end{array}$ & 50 & 0.026923 & 0.000498 \\
$\begin{array}{l}\text { Whole Dongbei long grain rice } \\
\text { and Meihe rice kernels }\end{array}$ & 100 & 0.028462 & 0.000689 \\
$\begin{array}{l}\text { Whole Dongbei long grain rice } \\
\text { and Thai jasmine rice kernels }\end{array}$ & 100 & 0.068235 & 0.000998 \\
$\begin{array}{l}\text { Whole Thai jasmine rice and } \\
\text { Meihe rice kernels }\end{array}$ & 100 & 0.078534 & 0.001521 \\
$\begin{array}{l}\text { Broken kernels } \\
\text { Whole and broken kernels }\end{array}$ & 50 & 0.080236 & 0.001598 \\
Whole and broken kernels & & & \\
\end{tabular}

Notes: *The comparison sample is the mixture of whole and broken kernels. **The comparison sample is a group of whole kernels and a group of broken kernels, which means that each whole kernel is compared with every broken kernel and each individual in the group of broken kernels is compared with every individual in the group of whole kernels.

We can see from table 2 that there are significant differences in similarity among different varieties of rice kernels as well as between whole rice kernels and broken rice kernels. Therefore, they can be classified and recognized in theory. We have used artificial neural network to recognize rice kernels with high efficiency.

\subsection{Recognition of Rice Kernels Using an Artificial Neural Network}

A BP neural network was used as the classifier. The network structure has three layers: an input layer, a hidden layer, and an output layer. There are nine nodes in the input layer, corresponding to the first nine RFD components. There are two nodes in the output layer, considering network expansibility. Suppose that an output of 00 denotes a standard rice kernel and an output of 11 denotes a broken kernel. Experimental tests revealed that there can be four hidden nodes, Tansig can be used for the activation function, and the YPROP algorithm can be used to deal with the problem of low convergence speed.

A sample of 200 rice kernels (150 whole and 50 broken) was used to train the network and then to determine the network weight value. Another sample of 800 rice kernels (600 whole and 200 broken) were used as test samples for classification. The test results are presented in Table 3. 
Table 3. Detection of broken rice using a BP neural network

\begin{tabular}{llll}
\hline Sample & Number & $\begin{array}{l}\text { Number } \\
\text { detected }\end{array}$ & Accuracy (\%) \\
\hline Whole Dongbei long grain rice kernels & 200 & 196 & 98 \\
Whole Meihe rice kernels & 200 & 192 & 96 \\
Whole Thai jasmine rice kernels & 200 & 200 & 100 \\
Broken rice kernels & 200 & 193 & 96.5 \\
\hline
\end{tabular}

\section{Conclusions}

In this research we developed an improved Fourier descriptor, a radius Fourier descriptor. Experimental test indicated that this RFD includes almost all the shape information of rice kernel. It is evident that we can calculate rice kernel area and differentiate rice variety and broken kernels using RFD. As a result, only using the first 9 RFD components we can reproduce rice contours without distortion and retain almost all of the feature information. Without concern for the initial starting point on the contour, this method is simple and the dimension of the feature vector can be reduced greatly. Taking China Dongbei long grain rice, Meihe rice, and Thai jasmine rice as examples, area of rice kernels and variety distance between different rice samples were calculated and a backpropagation neural network was tested for detection of broken rice kernels. The detection accuracy for whole rice kernels of different samples was $96 \%-100 \%$ and for broken rice kernels was $96.5 \%$. Combined with backpropagation neural network, we can classify rice variety accurately. Moreover, the RFD has the following advantages for describing the contours of rice kernels:

1. Uniqueness: the Fourier transform is a one-to-one mapping and one descriptor set corresponds to one boundary contour image.

2. Shift invariance: The transform coefficient of the RFD relies only on the boundary shape and is not related to the image position.

3. Rotation invariance: Image rotation only changes the phase spectrum of descriptor and the power spectrum does not change.

4. Scale invariance: The descriptor is scale-invariant after normalization.

5. Precise description: An image boundary can be totally restored using the RFD, which is impossible using other boundary description methods.

6. Low dimensionality of the feature vector: Fewer components are required to describe the boundary and the Euclidean distance is used for easy classification.

The RFD provides a means to analyze the shape of rice kernels in the frequency domain. It also provides a reference scheme for shape analysis of other agricultural products. For example, it has practical significance for quality assessment, classification and recognition of fruit, vegetables, seeds and other agricultural products.

Known limitations: when the RFD, which using amplitude spectrum of Fourier transform to describe rice shape, is used to do rice variety recognition, some preparation should be done in advance. If using variety distance, the average of 
standard rice kernels' descriptor should be calculated for statistics; if using artificial neural network, the standard sample should be used for training.

Acknowledgments. This research was partially supported by the Innovation and Technology Fund of Shandong Agricultural University of China, the Provincial Scholarship Fund of Shandong, China. The authors would like to thank the anonymous reviewers for the many useful observations that greatly contributed to improve the overall quality of the paper.

\section{References}

1. Ren, X.Z., Ma, X.Y.: Research advances of agricultural product grain shape identification and current situation of its application in the engineering field. Trans. Chin. Soc. Agric. Eng. 20(3), 276-280 (2004) (in Chinese)

2. Wang, F.J.: Rice Quality Detection Based on Digital Image Processing. Journal of Anhui Agricultural Sciences 38(22), 11998-11999, 12056 (2010) (in Chinese)

3. Shi, L.J., Wen, Y.X., Mou, T.M., Xu, J.Y.: Application Progress of Machine Vision Technology in Grain Detection. Hubei Agricultural Science 48(6), 1514-1518 (2009) (in Chinese)

4. Kilic, K., Boyaci, I.H., Koksel, H.: A classification system for beans using computer vision system and artificial neural networks. Journal of Food Engineering 78(3), 897-904 (2007)

5. Wang, B.T., Sun, J.A., Cai, A.N.: Relative moments and their applications to geometric shape recognition. Journal of Image and Graphics 6(3), 296-300 (2001) (in Chinese)

6. Nii, K., Kawabata, S.: Assessment of the Association between the Three-dimensional Shape of the Corolla and Two-dimensional Shapes of Petals Using Fourier Descriptors and Principal Component Analysis in Eustoma grandiflorum. Journal of the Japanese Society for Horticultural 80(2), 200-205 (2011)

7. Suzuki, K., Zheng, Z.Y., Tamura, Y.: Establishment of a Quantitative Evaluation Method of Rice Plant Type Using P-type Fourier Descriptors. Plant Production Science 14(2), 105$110(2011)$

8. Zhao, S.Q., Ding, W.M., Liu, D.Y.: Rice Hopper Shape Recognition Based on Fourier Descriptors. Transactions of the Chinese Society for Agricultural Machinery 40(8), 181184, 160 (2009)

9. Wei, Y., He, Y.W., Ni, H.F., Zhang, W.: Review on shape representation techniques and their applications in image retrieval. Systems Engineering and Electronics 31(7), 17551762 (2009)

10. Zhang, D.S., Lu, G.J.: Review of shape representation and description techniques. Pattern Recognition 37, 1-19 (2004)

11. Ling, Y., Wang, Y.M., Sun, M., Zhang, X.C.: Application of watershed algorithm to paddy image segmentation. Trans. Chin. Soc. Agric. Mach. 36(3), 95-98 (2005) (in Chinese)

12. Gao, H., Wang, Y.Q.: Study on the shape classification of farm produce based on computer vision. Comput. Eng. Appl. (14), 227-229 (2004) (in Chinese)

13. Wang, Y.Q., Gao, H.: Research on the feature description of similar round object. Computer Engineering 30(1), 158-169, 162 (2004) (in Chinese) 